\title{
EVALUACIÓN DE UN PROTOCOLO DE PROTECCIÓN VISUAL Y AUDITIVA EN ESTUDIANTES DE ODONTOLOGÍA
}

\author{
${ }^{1}$ Lizbeth Tatiana Cáceres Sierra, ${ }^{1}$ Isabel Teresa Pérez Sierra, ${ }^{1}$ Diana Carolina Arias Sarmiento, \\ ${ }^{2}$ Gloria Cristina Aránzazu Moya \\ ${ }^{1}$ Estudiante $\mathrm{X}$ semestre F. de Odontología U. Santo Tomás, Bucaramanga (Colombia) \\ ${ }^{2}$ Odontóloga U. Santo Tomás, Especialista en Patología y Medios Diagnósticos U. El Bosque, Docente U. Santo Tomás, Bucaramanga (Colombia)
}

Autor responsable de correspondencia: Gloria Cristina Aránzazu Moya

Correo electrónico: gloriacristina33@mail.ustabuca.edu.co

RESUMEN

Objetivo: diseñar y evaluar un protocolo de protección visual y auditiva para los estudiantes de odontología.

Materiales y métodos: se realizó un ensayo comunitario controlado con una muestra de 83 estudiantes, divididos en dos grupos (intervenido y control). Se aplicó a cada grupo una encuesta de conocimientos y una valoración de sus prácticas, una semana después se realizó la segunda observación y encuesta para el grupo control. Posteriormente, se intervino con el material educativo y se valoraron conocimientos y prácticas a este grupo. En el ánalisis univariado las variables se describieron mediante medidas de resumen según la naturaleza de cada una, como frecuencia, porcentaje, razón, limite superior e inferior. En el analisis bivariado se aplicaron pruebas estadísticas según la naturaleza de la variable, para las cualitativas se aplicó el test de Chi2 y test exacto de Fisher, para las cuantitativas t de Student.

Resultados: en la evaluación inicial los participantes del grupo control mostraron un conocimiento mayor que el grupo intervenido (4,3 $\pm 1,5$ vs. $5,2 \pm 1,8 \mathrm{p}=0,016)$, mientras que, en la evaluación final el puntaje de los dos grupos fue similar $(6,2 \pm 2,2$ vs. $6,5 \pm 2,3 \mathrm{p}=$ $6,00)$. El puntaje final del grupo intervenido fue mayor que el del grupo control sin existir diferencias significativas $(1,9 \pm 2,8$ vs. 1,2 $\pm 3,0 \mathrm{p}=0,296)$.

Conclusiones: no se obtuvo diferencias estadísticamente significativas entre la aplicación del manual y la mejoría de las prácticas, posiblemente debido a que la protección auditiva no es usada por ningún estudiante. [Cáceres LT, Pérez IT, Arias DC, Aránzazu GC. Evaluación de un protocolo de protección visual y auditiva en estudiantes de odontología. Ustasalud 2011; 10: 91 - 97 ]

Palabras clave: Visión, Escucha, Medidas preventivas.

\section{EVALUATION OF A PROTOCOL FOR VISUAL AND HEARING PROTECTION} IN DENTAL STUDENTS

\section{ABSTRACT}

Objective: to design and evaluate a protocol for eye and ear protection for dental students.

Methods: a controlled communitarian trail with a sample of 83 students was done. The sample was divided in two groups (experimental and control). A survey of knowledge and an assessment of practices were applied to each group; one week later, the survey was again applied to the control group. After that, the educational material was showed to the experimental group in order to establish knowledge and practice about visual and hearing protection. All the evaluations were done within a week interval. The variables were described using summary or dispersion measures. Chi2, Fisher Exact test or Student's t test were performed in the bivariate analyses. Results: at initial assessment, control group participants showed greater knowledge that the experimental group (4.3 \pm 1.5 vs. $5.2 \pm$ $1.8 \mathrm{p}=0.016)$, while in the final evaluation the two groups had a similar score $(6.2 \pm 2.2 \mathrm{vs} .6 .5 \pm 2.3 \mathrm{p}=6.00)$. The final score in the experimental group was higher than the control group but there were not significant differences between them (1.9 \pm 2.8 vs. 1.2 $\pm 3.0 \mathrm{p}=0.296)$.

Conclusions: there were no statistically significant differences between the application of the manual and the improvement of practices, possibly because any student did not use hearing protection.

Key words: Vision, Listening, Preventive measures. 


\section{INTRODUCCIÓN}

Es importante conocer claramente los efectos que tiene sobre la salud del odontólogo el no uso o uso incorrecto de protección ocular y auditiva. Entre estos efectos se encuentran las lesiones oculares que van desde la fatiga ocular, infecciones, cuerpos extraños que se introducen en el ojo, hasta abrasiones de la cornea, en el campo auditivo se presentan lesiones tales como el tinnitus, la sordera profesional y otra secuelas como stress y dolor de cabeza, entre otros. ${ }^{1}$

Actualmente, los profesionales de odontología tienen conocimiento de los efectos y problemas que trae la falta de protección ocular y aun así, muchos profesionales no la usan o la usan de manera incorrecta y no la proporcionan a sus pacientes. ${ }^{1,2}$

La odontología preventiva en el campo de la salud ocupacional busca que el odontólogo sea consciente de la necesidad de usar la protección ocular, pese a esto, estudios realizados anteriormente, han encontrado que el $10 \%$ de las lesiones que presentan los odontólogos son oculares y que el $50 \%$ de los odontólogos no usan la protección ocular al momento de ocurrir el evento. ${ }^{3-5}$ Es por ello, que el desconocimiento de los síntomas básicos de las lesiones oculares, la falta de una protección correcta y la ausencia de normas escritas de protección son factores de riesgo que aumentan la posibilidad de sufrir alguna lesión ocular entre el personal de odontología. ${ }^{6} \mathrm{Al}-$ gunas de las lesiones oculares más frecuentes son cuerpos extraños e infecciones oculares, abrasiones de la cornea, quemadura o daño por productos químicos y fatiga ocular. ${ }^{1,3,47-12}$ En el campo auditivo es importante saber que el oído es el órgano sensorial responsable de la audición y el equilibrio. Se divide en tres zonas: externa, media e interna. ${ }^{13}$ La audición, es uno de los cinco sentidos principales, por el cual el oído, percibe las ondas de sonido. Según el National Institute for Ocupational Safety and Health si el oído se expone a $85 \mathrm{db}$ debe ser menos de 8 horas diarias, si se expone a $90 \mathrm{db}$ máximo ocho horas, de $95 \mathrm{db}$ solo cuatro horas y $115 \mathrm{db}$ en quince minutos. ${ }^{1,13,14}$

El profesional de la odontología está propenso a presentar diversos trastornos auditivos relacionados con el ejercicio laboral, el cual incluye varios factores de riesgo; como lo son el ruido producido por la pieza de mano, elementos rotatorios, turbinas, motores de laboratorio, eyector y el ruido general de consultorio, se llegan a niveles de ruido hasta de $101 \mathrm{db}$; pueden ocasionar sordera profesional, tinnitus, dolores de cabeza, stress, pulso acelerado, aumento de la presión arterial, nerviosismo, fatiga mental, frustraciones emocionales, aislamiento so- cial, deterioro de la comunicación con la sociedad, pérdida de productividad, interferencia en el sueño e interferencia en la concentración, entre otros; estos hallazgos sugieren que los niveles de ruido significativos en el entorno de la salud oral pueden perjudicar la calidad de vida de los profesionales y acelerar el deterioro del aparato auditivo, estos efectos se producen por altos niveles de ruidos, según la intensidad, distancia de la fuente y duración de exposición, se afecta así el desempeño y la habilidad del profesional para realizar con calidad los procedimientos odontológicos correctos y los momentos de la práctica donde debe ser usada dicha protección. El objetivo de este estudio fue diseñar un protocolo de protección visual y auditiva dirigido a los estudiantes de las clínicas odontológicas de la Universidad Santo Tomás.

\section{MATERIALES Y MÉTODOS}

Se realizó un ensayo comunitario controlado, con una muestra 83 estudiantes de sexto semestre quienes se dividieron aleatoriamente en dos grupos (intervenido y control). Se aplicaron dos instrumentos, uno de trece variables de tipo sociodemográfico, protección visual y auditiva y desinfección que fue respondido por los estudiantes (instrumento de conocimientos) y otro de observación con nueve variables de uso y desinfección de la protección visual y auditiva, que fue diligenciado por los investigadores (instrumento de observación). Se realizó el ejercicio de observación y se diligenció el instrumento por los investigadores. Una vez realizada la observación se entregó a los dos grupos control e intervenido el instrumento de conocimientos que fue diligenciado. En la siguiente sesión se repitió el ejercicio de observación con el grupo control y de nuevo se les dio el instrumento de conocimientos.

Una vez aplicado el segundo instrumento de prácticas y conocimientos al grupo control se entregó el protocolo de protección visual y auditiva al grupo intervenido para reducir sesgos de información.

En la siguiente sesión se repitió el ejercicio de observación con el grupo intervenido, una vez listo el instrumento de observación, se les dio el instrumento de conocimientos el cual diligenciaron. Cada actividad se realizó con una semana de diferencia. Todos los procedimientos fueron explicados a los participantes, se definieron los objetivos, riesgos, beneficios y garantías y la persona libremente pudo decidir si se vinculaba a la investigación.

\section{Análisis estadístico}

La información se proceso a través de una base de datos en excel y se analizó a través del sofware Stata 9,0. En el ánalisis univariado las variables se 
describieron mediante medidas de resumen según la naturaleza de cada una, como frecuencia, porcentaje, razón, límite superior e inferior. En el análisis bivariado se aplicaron pruebas estadísticas según la naturaleza de la variable, para las cualitativas se aplicó el test de $\mathrm{Chi}^{2}$ y test exacto de Fisher, para las cuantitativas t de Student.

\section{RESULTADOS}

La muestra inicialmente estuvo conformada por 58 mujeres y 29 hombres, estudiantes de sexto semestre de la facultad de odontología de la universidad Santo Tomás. El análisis de la evaluación inicial no mostró diferencias estadísticamente significativas en las prácticas de protección visual por ítem, salvo en la pregunta, proporciona protección visual al paciente, en la cual un porcentaje mayor de estudiantes del grupo control $(36,6 \%)$ proporciona siempre protección visual al paciente comparado con el grupo intervenido (10,9\%). Cabe resaltar que los participantes no usaban protector auditivo y, por lo tanto, no lo limpiaban ni desinfectaban (Tabla 1 y 2). De los 87 participantes que inicialmente fueron evaluados 83 respondieron nuevamente la encuesta, en la evaluación final.

Al igual que en la evaluación inicial, se encontraron diferencias estadísticamente significativas en la variable "proporciona protección visual al paciente". En las demás variables no se encontraron diferencias estadísticamente significativas entre las prácticas de protección visual y auditiva del grupo control e intervenido. En variables como uso de protección, pasó de $50 \%$ a $80 \%$ que reportan usarla siempre; la variable limpia el protector visual, pasó de $47 \%$ a $71 \%$ que los limpian siempre. No se observaron diferencias estadísticamente significativas al comparar las prácticas antes y después de la intervención en el grupo intervenido y el grupo control.

En la Tabla 3 se presentan los puntajes globales de prácticas de protección visual y auditiva. En la evaluación inicial los participantes del grupo control mostraron un puntaje mayor comparado con el grupo intervenido $(4,3 \pm 1,5$ vs. $5,2 \pm 1,8 \mathrm{p}=0,016)$, mientras que, en la evaluación final el puntaje de los dos grupos fue similar $(6,2 \pm 2,2$ vs. $6,5 \pm 2,3$ $\mathrm{p}=6,00$ ). Al analizar la evaluación inicial y final no se encontraron diferencias estadísticamente significativas por grupo, sin embargo, el puntaje final del grupo intervenido fue mayor que el del grupo control $(1,9 \pm 2,8$ vs. $1,2 \pm 3,0 \mathrm{p}=0,296)$.

En la Tabla 4 se presenta el riesgo para mejorar las prácticas de protección visual y auditiva. Se consideró que mejoraron las prácticas de protección si el puntaje final superó el puntaje inicial, no mejoraron las prácticas de protección si el puntaje inicial fue igual o el puntaje final fue inferior al puntaje inicial. Se encontró que la probabilidad de mejorar las prácticas de protección fue de 1,03 (IC95\% 0,72-1,48) veces en el grupo intervenido comparado con el grupo control, como el intervalo de confianza pasa por el valor nulo no se encontró asociación entre la intervención y la mejoría de prácticas de protección visual y auditiva en los estudiantes de odontología.

En la prueba de observación se encontró que los datos recogidos por los investigadores coincidían con lo reportado por los estudiantes en la mayoría de las variables, notándose una leve mejoría en el grupo intervenido en cuanto a la variable de limpieza de los protectores visuales y en otras como la variable desinfección, se encontró que los estudiantes reportaron sí desinfectar los protectores visuales pero en la prueba de observación se evidenció que inicialmente el $100 \%$ de grupo intervenido y el $99,6 \%$ del grupo control no lo hacían, después de la intervención se reportó una mejoría pero aún no coincide con lo reportado por los estudiantes, los porcentajes observados son menores a los reportados (Tabla 5). En la variable proporciona protección visual al paciente, el grupo intervenido mostró mejores porcentajes que el control antes y después de la intervención según lo observado por los investigadores.

\section{DISCUSIÓN}

En el presente estudio realizado en los estudiantes de sexto semestre de la Universidad Santo Tomás no se encontró una diferencia estadísticamente significativa entre la intervención, es decir, la implementación del protocolo y la mejoría de las prácticas clínicas, pese a que el grupo intervenido mostró un $1,9 \%$ de mejoría en las prácticas frente a un 1,2\% del grupo control. Se encontró también que el 100\% de los estudiantes de ambos grupos no usan protección auditiva. La falta de asociación y de uso de protección auditiva puede deberse a que no existe una norma en las clínicas odontologías de la Universidad Santo Tomás que motive a los estudiantes a usar protección auditiva ni una cátedra que enseñe la importancia de ésta, por lo que a pesar de la implementación del protocolo, no se evidenció ningún cambio en este aspecto. Garbín y colaboradores realizaron un estudio en 80 estudiantes de odontología de la Universidad de Central de Venezuela, encontraron que no es obligatorio la utilización de protectores auditivos al tener en cuenta el nivel de ruido producido en la clínica que era máximo de 83,4 decibeles, se encontró también que los niveles de ruido someten al profesional a un aumento del estrés y de la incomodidad al trabajar, por lo que recomiendan que el profesional debe adoptar medidas preventivas durante su trabajo usando 
Tabla 1. Prácticas de protección visual antes y después de la intervención según grupos de comparación.

\begin{tabular}{|c|c|c|c|c|c|c|c|c|c|c|}
\hline \multirow{3}{*}{ Variable } & \multicolumn{4}{|c|}{ Grupo intervenido $(n=45)$} & \multicolumn{6}{|c|}{ Grupo control $(n=38)$} \\
\hline & \multicolumn{2}{|c|}{ Antes } & \multicolumn{2}{|c|}{ Después } & \multirow[t]{2}{*}{$\mathbf{P}$} & \multicolumn{2}{|c|}{ Antes } & \multicolumn{2}{|c|}{ Después } & \multirow[t]{2}{*}{$\mathbf{P}$} \\
\hline & Fc & $(\%)$ & Fc & $(\%)$ & & Fc & $(\%)$ & FC & $(\%)$ & \\
\hline \multicolumn{11}{|l|}{ Uso de protección visual } \\
\hline Siempre & 23 & 51,1 & 36 & 80,0 & $1,000^{*}$ & 22 & 57,9 & 33 & 86,8 & 0,457 \\
\hline Algunas veces & 16 & 35,6 & 3 & 6,7 & & 14 & 36,8 & 4 & 10,5 & \\
\hline Frecuentemente & 5 & 11,1 & 6 & 13,3 & & 2 & 5,3 & 1 & 2,6 & \\
\hline Casi nunca & 1 & 2,2 & 0 & 0,0 & & 0 & 0,0 & 0 & 0,0 & \\
\hline Nunca & 0 & 0,0 & 0 & 0,0 & & 0 & 0,0 & 0 & 0,0 & \\
\hline \multicolumn{11}{|l|}{ Tipo de protección visual } \\
\hline Gafas de fórmula & 4 & 8,9 & 3 & 6,7 & $0,942^{*}$ & 2 & 5,3 & 1 & 2,6 & $0,579^{\prime}$ \\
\hline Gafas de protección & 18 & 40,0 & 5 & 11,1 & & 8 & 21,1 & 4 & 10,5 & \\
\hline Gafas con protección lateral & 2 & 4,4 & 6 & 13,3 & & 2 & 5,3 & 4 & 10,5 & \\
\hline Visor & 21 & 46,7 & 31 & 68,9 & & 26 & 68,4 & 29 & 76,3 & \\
\hline \multicolumn{11}{|c|}{ Procedimiento en que utiliza protección visual } \\
\hline Periodoncia & 0 & 0,0 & 1 & 2,2 & $1,000^{*}$ & 1 & 2,6 & 0 & 0,0 & $1,000^{*}$ \\
\hline Cirugía & 1 & 2,2 & 1 & 2,2 & & 1 & 2,6 & 0 & 0,0 & \\
\hline Endodoncia & 0 & 0,0 & 0 & 0,0 & & 0 & 0,0 & 0 & 0,0 & \\
\hline Examen clínico & 0 & 0,0 & 0 & 0,0 & & 0 & 0,0 & 0 & 0,0 & \\
\hline Operatoria & 1 & 2,2 & 1 & 2,2 & & 3 & 7,9 & 2 & 7,3 & \\
\hline Todas las anteriores & 43 & 95,6 & 42 & 93,3 & & 34 & 89,5 & 36 & 87,9 & \\
\hline \multicolumn{11}{|c|}{ Proporciona protección visual al paciente } \\
\hline Siempre & 5 & 11,1 & 24 & 53,3 & $0,251^{*}$ & 15 & 39,5 & 28 & 73,7 & $0,844^{*}$ \\
\hline Algunas veces & 14 & 31,1 & 13 & 28,9 & & 15 & 39,5 & 3 & 7,9 & \\
\hline Frecuentemente & 6 & 13,3 & 2 & 4,4 & & 1 & 2,6 & 5 & 13,2 & \\
\hline Casi nunca & 12 & 26,7 & 2 & 4,4 & & 2 & 5,3 & 1 & 2,6 & \\
\hline Nunca & 8 & 17,8 & 4 & 8,9 & & 5 & 13,2 & 1 & 2,6 & \\
\hline \multicolumn{11}{|l|}{ Limpia el protector visual } \\
\hline Siempre & 20 & 44,4 & 32 & 71,1 & $0,360^{*}$ & 22 & 57,9 & 26 & 68,4 & $0,278^{*}$ \\
\hline Algunas veces & 18 & 40,0 & 6 & 13,3 & & 12 & 31,6 & 8 & 21,1 & \\
\hline Frecuentemente & 3 & 6,7 & 3 & 6,7 & & 2 & 5,3 & 0 & 0,0 & \\
\hline Casi nunca & 3 & 6,7 & 1 & 2,2 & & 2 & 5,3 & 3 & 7,9 & \\
\hline Nunca & 1 & 2,2 & 3 & 6,7 & & 0 & 0,0 & 1 & 2,6 & \\
\hline \multicolumn{11}{|l|}{ Desinfecta el protector visual } \\
\hline Siempre & 8 & 17,8 & 26 & 57,8 & $0,169^{*}$ & 12 & 31,6 & 21 & 55,3 & $0,185^{*}$ \\
\hline Algunas veces & 20 & 44,4 & 7 & 15,6 & & 8 & 21,1 & 9 & 23,7 & \\
\hline Frecuentemente & 3 & 6,7 & 6 & 13,3 & & 5 & 13,2 & 1 & 2,6 & \\
\hline Casi nunca & 6 & 13,3 & 1 & 2,2 & & 6 & 15,8 & 5 & 13,2 & \\
\hline Nunca & 8 & 17,8 & 5 & 11,1 & & 7 & 18,4 & 2 & 5,3 & \\
\hline \multicolumn{11}{|c|}{ Accidentes en prácticas clínicas en sus ojos } \\
\hline Siempre & 0 & 0,0 & 1 & 2,2 & $0,081^{* *}$ & 1 & 2,6 & 1 & 2,6 & $0,441^{*}$ \\
\hline Algunas veces & 4 & 8,9 & 6 & 13,3 & & 1 & 2,6 & 2 & 5,3 & \\
\hline Frecuentemente & 0 & 0,0 & 1 & 2,2 & & 0 & 0,0 & 2 & 5,3 & \\
\hline Casi nunca & 9 & 20,0 & 6 & 13,3 & & 2 & 5,3 & 8 & 21,1 & \\
\hline Nunca & 32 & 71,1 & 31 & 68,9 & & 34 & 89,5 & 25 & 65,8 & \\
\hline
\end{tabular}


Tabla 2. Prácticas de protección auditiva antes y después de la intervención según grupos de comparación

\begin{tabular}{|c|c|c|c|c|c|c|c|c|c|c|}
\hline \multirow{3}{*}{ Variable } & \multicolumn{4}{|c|}{ Grupo intervenido $(n=45)$} & \multicolumn{6}{|c|}{ Grupo control $(n=38)$} \\
\hline & \multicolumn{2}{|c|}{ Antes } & \multicolumn{2}{|c|}{ Después } & \multirow[t]{2}{*}{$\mathbf{P}$} & \multicolumn{2}{|c|}{ Antes } & \multicolumn{2}{|c|}{ Después } & \multirow[t]{2}{*}{$\mathbf{P}$} \\
\hline & Fc & $(\%)$ & Fc & $(\%)$ & & Fc & $(\%)$ & FC & $(\%)$ & \\
\hline \multicolumn{11}{|c|}{ Uso de protección auditiva } \\
\hline Siempre & 0 & 0,0 & 3 & 6,6 & $1,000^{*}$ & 0 & 0,0 & 2 & 5,3 & NA \\
\hline Algunas veces & 0 & 0,0 & 1 & 2,2 & & 0 & 0,0 & 1 & 1,27 & \\
\hline Frecuentemente & 0 & 0,0 & 0 & 0,0 & & 0 & 0,0 & 0 & 0,0 & \\
\hline Casi nunca & 3 & 6,7 & 0 & 0,0 & & 0 & 0,0 & 1 & 2,7 & \\
\hline Nunca & 42 & 93,3 & 41 & 91,1 & & 38 & 100 & 34 & 89,5 & \\
\hline \multicolumn{11}{|c|}{ Tipo de protección auditivo } \\
\hline Tapones & 0 & 0,0 & 3 & 6,7 & NA & 0 & 0,0 & 3 & 7,9 & NA \\
\hline Orejeras & 0 & 0,0 & 0 & 0,0 & & 0 & 0,0 & 0 & 0,0 & \\
\hline No usa & 46 & 100 & 42 & 93,3 & & 38 & 100 & 35 & 92,1 & \\
\hline \multicolumn{11}{|c|}{ Limpia y desinfecta su protector auditivo } \\
\hline Siempre & 0 & 0,0 & 3 & 6,7 & NA & 0 & 0,0 & 2 & 5,3 & NA \\
\hline Algunas veces & 0 & 0,0 & 0 & 0,0 & & 0 & 0,0 & 1 & 2,6 & \\
\hline Frecuentemente & 0 & 0,0 & 0 & 0,0 & & 0 & 0,0 & 0 & 0,0 & \\
\hline Casi nunca & 0 & 0,0 & 1 & 2,2 & & 0 & 0,0 & 0 & 0,0 & \\
\hline Nunca & 46 & 100 & 41 & 91,1 & & 38 & 100 & 35 & 92,1 & \\
\hline \multicolumn{11}{|c|}{ Manifiesta molestias auditivas después de sus prácticas clínicas } \\
\hline Siempre & 1 & 2,2 & 0 & 0,0 & 0,436 & 1 & 2,2 & 0 & 0,0 & 0,794 \\
\hline Algunas veces & 4 & 8,9 & 10 & 22,2 & & 4 & 8,9 & 10 & 22,2 & \\
\hline Frecuentemente & 0 & 0,0 & 1 & 2,2 & & 0 & 0,0 & 1 & 2,2 & \\
\hline Casi nunca & 9 & 20,0 & 7 & 15,6 & & 9 & 20,0 & 7 & 15,6 & \\
\hline Nunca & 31 & 68,9 & 27 & 60,0 & & 31 & 68,9 & 27 & 60,0 & \\
\hline \multicolumn{11}{|c|}{ Sustancias que usa para la desinfección de protectores visuales } \\
\hline Glutaraldehído & 9 & 20,0 & 15 & 33,3 & 0,413 & 8 & 21,1 & 13 & 34,2 & 0,351 \\
\hline Hipoclorito & 11 & 24,4 & 6 & 13,3 & & 7 & 18,4 & 5 & 13,2 & \\
\hline Clorexidina & 1 & 2,2 & 0 & 0,0 & & 0 & 0,0 & 1 & 2,6 & \\
\hline Otras & 7 & 15,7 & 19 & 42,2 & & 10 & 26,3 & 14 & 36,8 & \\
\hline No usa & 17 & 37,8 & 5 & 11,1 & & 13 & 34,2 & 5 & 13,2 & \\
\hline \multicolumn{11}{|c|}{ Sustancias que usa para la desinfección de protectores auditivos } \\
\hline Glutaraldehído & 1 & 2,2 & 2 & 4,4 & $1,000^{*}$ & 1 & 2,6 & 2 & 5,3 & $0,105^{*}$ \\
\hline Hipoclorito & 0 & 0,0 & 0 & 0,0 & & 0 & 0,0 & 0 & 0,0 & \\
\hline Clorexidina & 0 & 0,0 & 0 & 0,0 & & 0 & 0,0 & 0 & 0,0 & \\
\hline Otras & 0 & 0,0 & 1 & 2,2 & & 0 & 0,0 & 2 & 5,3 & \\
\hline No usa & 44 & 97,8 & 42 & 93,3 & & 37 & 97,4 & 34 & 89,5 & \\
\hline
\end{tabular}

Test Exacto de Fisher

Tabla 3. Comparación de puntaje de prácticas de protección visual y auditiva según prueba inicial y final

\begin{tabular}{|c|c|c|c|c|c|c|c|}
\hline Variable & $\begin{array}{l}\text { Puntaje } \\
\text { inicial* }\end{array}$ & $\mathbf{P}$ & $\begin{array}{c}\text { Puntaje } \\
\text { Final }^{*}\end{array}$ & $\mathbf{P}$ & $\begin{array}{l}\text { Diferencia } \\
\text { puntajes* }\end{array}$ & IC95\% & $\mathbf{P}$ \\
\hline \multicolumn{8}{|l|}{ Grupo } \\
\hline Intervenido & $4,3 \pm 1,5$ & 0,016 & $6,2 \pm 2,2$ & 0,600 & $1,9 \pm 2,8$ & $0,24 \quad 2,22$ & 0,296 \\
\hline Control & $5,2 \pm 1,8$ & & $6,5 \pm 2,3$ & & $1,2 \pm 3,0$ & $1,06 \quad 2,76$ & \\
\hline
\end{tabular}

* Promedio \pm desviación estándar. IC95\%: intervalo de confianza (95\%). Prueba t de Student. 
Tabla 4. Cálculo de riesgo relativo para mejorar las prácticas de protección visual y auditiva en estudiantes de sexto semestre de la Universidad Santo Tomás, 2010

\begin{tabular}{|c|c|c|c|c|c|c|c|}
\hline \multirow{3}{*}{$\begin{array}{l}\quad \text { Variable } \\
\text { Grupo } \\
\text { Intervenido }\end{array}$} & \multirow{2}{*}{ Global } & \multirow[t]{2}{*}{$\mathrm{Si}$} & \multirow[t]{2}{*}{ No } & \multirow[t]{2}{*}{ RR crudo } & \multicolumn{2}{|c|}{ IC95\% } & \multirow[t]{2}{*}{$\mathbf{P}$} \\
\hline & & & & & & & \\
\hline & 45 & 27 & 18 & 1,030 & 0,720 & 1,480 & 0,845 \\
\hline Control & 38 & 22 & 16 & & & & \\
\hline
\end{tabular}

$\mathrm{Chi}^{2}$

Tabla 5. Distribución por frecuencias y porcentaje de prácticas del grupo intervenido y control antes y después

\begin{tabular}{|c|c|c|c|c|c|c|c|c|}
\hline \multirow{3}{*}{ Variable } & \multicolumn{2}{|c|}{ Intervenido 1} & \multicolumn{2}{|c|}{ Intervenido 2} & \multicolumn{2}{|c|}{ Control 1} & \multicolumn{2}{|c|}{ Control 2} \\
\hline & \multicolumn{4}{|c|}{$n=46$} & \multicolumn{4}{|c|}{$\mathrm{n}=41$} \\
\hline & Fc & $\%$ & Fc & $\%$ & Fc & $\%$ & FC & $\%$ \\
\hline \multicolumn{9}{|c|}{ Procedimiento que realiza el estudiante } \\
\hline Periodoncia & 9 & 19,6 & 0 & 0 & 1 & 2,4 & 1 & 2,4 \\
\hline Cirugía & 10 & 21,7 & 0 & 0 & 0 & 0 & 0 & 0 \\
\hline Endodoncia & 2 & 4,3 & 0 & 0 & 1 & 2,4 & 3 & 5 \\
\hline Operatoria & 16 & 34,8 & 28 & 61,4 & 22 & 52 & 23 & 56,1 \\
\hline Rehabilitación & 0 & 0 & 0 & 0 & 0 & 0 & 1 & 2,4 \\
\hline Examen clínico & 9 & 19,3 & 9 & 19,3 & 17 & 40 & 2 & 4,9 \\
\hline Sin paciente & 0 & 0 & 9 & 19,3 & 0 & 0 & 11 & 58 \\
\hline \multicolumn{9}{|l|}{ Uso de protección visual } \\
\hline $\mathrm{Si}$ & 23 & 50 & 27 & 54 & 14 & 33 & 19 & 44,9 \\
\hline No & 23 & 50 & 10 & 20 & 27 & 67.3 & 11 & 28 \\
\hline \multicolumn{9}{|c|}{ Tipo de protección que usa el estudiante } \\
\hline Gafas de fórmula & 0 & 0 & 4 & 8,7 & 0 & 0 & 2 & 4,9 \\
\hline Gafas de protección & 0 & 0 & 0 & 0 & 0 & 0 & 1 & 2,4 \\
\hline Gafas con protectores laterales & 7 & 15,2 & 4 & 8,7 & 10 & 24,4 & 4 & 8,3 \\
\hline Visor & 16 & 34,8 & 19 & 41,3 & 4 & 9,1 & 12 & 29,3 \\
\hline \multicolumn{9}{|c|}{ Suministra protección visual al paciente } \\
\hline $\mathrm{Si}$ & 1 & 2,2 & 13 & 27 & 2 & 4,9 & 15 & 36,6 \\
\hline No & 45 & 97 & 24 & 51 & 17 & 40 & 15 & 36,6 \\
\hline \multicolumn{9}{|l|}{ Limpieza del protector visual } \\
\hline $\mathrm{Si}$ & 0 & 0 & 11 & 23,9 & 1 & 2,4 & 11 & 28 \\
\hline No & 24 & 51 & 11 & 23,9 & 27 & 67,3 & 24 & 60 \\
\hline \multicolumn{9}{|l|}{ Desinfección del protector visual } \\
\hline $\mathrm{Si}$ & 0 & 0 & 11 & 23,9 & 1 & 2,4 & 11 & 28 \\
\hline No & 46 & 100 & 26 & 53 & 40 & 99,6 & 19 & 44,9 \\
\hline \multicolumn{9}{|c|}{ Uso de elementos rotatorios o que emitan ruidos } \\
\hline $\mathrm{Si}$ & 16 & 34,8 & 27 & 54 & 36 & 87,8 & 24 & 60 \\
\hline No & 30 & 65,5 & 10 & 20 & 5 & 12,2 & 6 & 14,6 \\
\hline \multicolumn{9}{|l|}{ Uso de protector auditivo } \\
\hline $\mathrm{Si}$ & 0 & 0 & 0 & 0 & 0 & 0 & 0 & 0 \\
\hline No & 46 & 100 & 37 & 74,4 & 41 & 100 & 30 & 73,2 \\
\hline \multicolumn{9}{|c|}{ Sustancia de desinfección de protectores visuales y auditivos } \\
\hline Glutaraldehído & 20 & 44 & 16 & 34 & 10 & 24,4 & 6 & 14,6 \\
\hline Hipoclorito & 2 & 4,3 & 0 & 0 & 5 & 12,2 & 1 & 2,4 \\
\hline Clorhexidina & 0 & 0 & 0 & 0 & 0 & 0 & 0 & 0 \\
\hline Otros & 0 & 0 & 9 & 19,3 & 1 & 2,4 & 1 & 2,4 \\
\hline
\end{tabular}


los protectores auditivos para evitar lesiones a lo largo de su carrera. ${ }^{15}$

De otra parte, en un estudio realizado en 48 docentes odontólogos encontraron que el $29 \%$ reportó presentar dolor de cabeza después de la exposición al ruido de la clínica. ${ }^{7}$ En el presente estudio se observó que el 19,5\% del grupo intervenido y el 10\% del grupo control tenía alguna molestia como dolor de cabeza después de salir de las clínicas, lo que evidencia la falta del uso preventivo del protector auditivo. Rivera y colaboradores encontraron que según la escala Elí las mujeres evidenciaron alteración en un $62 \%$ en el oído izquierdo y los hombres en la escala de Larsen una alteración en el oído derecho del $88.9 \%$, lo que demuestra la afección de la audición por el no uso de los protectores auditivos. ${ }^{1}$

En cuanto al uso de protección visual se encontró que el 56\% del grupo control y el 50\% del grupo intervenido usaba siempre el protector ocular, de estos el $28 \%$ y $21 \%$ reportaron usar visor. Farrier y colaboradores reportaron que el $94 \%$ de los odontólogos evaluados eran conscientes de la importancia de la protección ocular y el $87 \%$ usaban alguna forma de protección, de estos el 58\% usaban gafas personales que no eran adecuadas y el $65 \%$ no contaba con un protocolo escrito en cuanto a dicha protección. ${ }^{2}$

Después de la aplicación del protocolo, el grupo intervenido mostró un aumento en la variables usa protección visual que pasó del $50 \%$ al $63 \%$, en proporción a protección visual al paciente donde del $10,9 \%$ pasó al $53,3 \%$, las variables de limpieza y desinfección también tuvo un aumento significativo al pasar de $45,7 \%$ y $17,4 \%$ respectivamente a $71,1 \%$ y $57,8 \%$, en las variables de protección auditiva no se encontró ningún cambio significativo, se comprobó así que los estudiantes entienden la importancia de usar protectores visuales como se evidenció en los estudios realizado por Farrier y colaboradores, ${ }^{2}$ pero no comprenden la importancia de la prevención en el campo auditivo.

Cabe resaltar que este es el primer protocolo que se implementa en las clínicas que explique de manera clara la forma de uso, lavado y desinfección de los protectores visuales y auditivos.

\section{Conclusiones}

Pese al nivel de conocimientos que poseen los estudiantes acerca del uso de protección visual muchos de ellos no tienen claridad de cómo se debe lavar y desinfectar los protectores visuales y auditivos.

Ningún estudiante de VI semestre de la Universidad Santo Tomás hace uso preventivo de protección auditiva.

\section{BIBLIOGRAFÍA}

1. Rivera YL, Rueda SJ, Concha SC. Pérdida auditiva inducida por ruido evaluada en odontólogos docentes de las clínicas odontológicas de la Universidad Santo Tomás. Ustasalud 2007; 6: 96 - 103

2. Farrier SL, Farrier JN, Gilmour AS. Eye safety in operative dentistry - A study in general dental practice. Br Dent J 2006; 200: 218 - 223

3. Nakagawara VB. Functional model of an eye protection program: guide for the clinical optometrist. J Am Optom Assoc 1988; 59: 925 - 928

4. Zárate AM, Rezzonico MS, Castillo MC, Castillo G, Castillo B, Bregains L, et al. Bioseguridad e higiene en la formación del odontólogo. Acta Odontol Venez [en línea] 2009; 47 (1): 102 - 109 URL disponible en: http://www. scielo.org.ve/scielo.php?script =sci_arttext\&pid = S000163652009000100013\&lng $=$ es\&nrm $=$ iso

5. Bruzell REM, Jacobsen N, Hensten PA. Health hazards associated with curing light in the dental clinic. Clin Oral Invest 2004; 8: 113 - 117

6. Chadwick RG, Alatsaris M, Ranka M. Eye care habits of dentist registered in the United Kingdom. Br Dent J 2007; 203: E7

7. Golding JM, Franceshi T. Pérdida gradual de la audición causada por el ruido emitido en el consultorio dental. [en línea]. URL disponible en: http://odontologia-vital.ulatina.ac.cr/PerdidaAudicion.pdf

8. Troconis JE. Control del ambiente de los consultorios odontológicos: uso de gorro, máscara de larga cobertura, bata quirúrgica, dique de goma y guantes [en línea]. URL disponible en: http://www.opas.org.br/gentequefazsaude/bvsde/bvsacd/cd49/guantes.pdf

9. Gijbels F, Jacobs R, Princen K, Nackaerts O, Debruyne F. Potential occupational health problems for dentists in Flanders, Belgium. Clin Oral Invest 2006; 10: 8 - 16

10. Ward MA, Compliance with infection control precautions in primary care. Primary Health Care 2006; 16: 35-39

11. Peate WF. Work-related eye injuries and illnesses. Am Fam Physician 2007; 75: 1017 - 1022

12. Leggat PA, Kendjarune U, Smith DR. Occupational health problems in modern dentistry: a review. Industrial Health 2007; 45: 611- 621

13. Hill EE. Eye safety practices in U.S dental school restorative clinics, 2006. J Dent Edu. 2006; 70: 1294 - 1297

14. Rekus JF. The eyes have it. Occupational Hazards 2001; 63: $48-52$

15. Garvin AJI, Garvin CAS, Ferreira NF, Ferreira NL. Evaluación de la incomodidad ocupacional: nivel del ruido de una clínica de graduación. Acta Odontol Venez 2006; 44 (1) [en línea]. URL disponible en: http://www.actaodontologica.com/ediciones/2006/1/incomodidad_ocupacional.asp

\section{Correos electrónicos de los autores:}

Lizbeth Tatiana Cáceres Sierra: liztacasi@ @hotmail.com Isabel Teresa Pérez Sierra: isabelescondy@hotmail.com Diana Carolina Arias Sarmiento: carolina_88_272@hotmail.com Gloria Cristina Aránzazu M.: gloriacristina33@mail.ustabuca.edu.co 